\title{
Chiral nucleon-nucleon forces in nuclear structure calculations
}

\author{
L. Coraggio ${ }^{1}$, A. Gargano ${ }^{1}$, J. W. Holt $^{2}$, N. Itaco ${ }^{1}$, R. \\ MACHLEIDT $^{3}$, L. E. MARCUCCI ${ }^{4}$, and F. SAMmARRUCA ${ }^{3}$
}

${ }^{1}$ INFN, Sezione di Napoli, Via Cintia - I-80126 Napoli, Italy

2 Physics Department, University of Washington, Seattle, WA 98195, USA

${ }^{3}$ Department of Physics, University of Idaho, Moscow, ID 83844, USA

${ }^{4}$ Dipartimento di Fisica "Enrico Fermi", Università di Pisa, and INFN, Sezione di Pisa, Largo B. Pontecorvo 3 - I-56127 Pisa, Italy

\begin{abstract}
Realistic nuclear potentials, derived within chiral perturbation theory, are a major breakthrough in modern nuclear structure theory, since they provide a direct link between nuclear physics and its underlying theory, namely the QCD. As a matter of fact, chiral potentials are tailored on the low-energy regime of nuclear structure physics, and chiral perturbation theory provides on the same footing two-nucleon forces as well as many-body ones. This feature fits well with modern advances in ab-initio methods and realistic shell-model. Here, we will review recent nuclear structure calculations, based on realistic chiral potentials, for both finite nuclei and infinite nuclear matter.
\end{abstract}

\section{Introduction}

The last decade has seen chiral EFT emerging as a powerful tool to describe the interaction among hadrons in a low-energy regime in a systematic and model-independent way (see Refs. [1,2] for recent reviews).

Generally, in nuclear many-body systems the nuclear momenta are on the order of the pion mass, so the construction of chiral nuclear potentials is based on an expansion in powers of this soft scale $\left(Q \sim m_{\pi}\right)$ over the 
hard scale, which is set by the typical hadron masses $\Lambda_{\chi} \sim m_{\rho} \sim 1 \mathrm{GeV}$. The latter is also known as the chiral-symmetry breaking scale. The EFT must have a firm link with quantum chromodynamics (QCD) so to emerge above the level of phenomenology. This is obtained having the EFT undergo all relevant symmetries of the underlying theory, in particular, the broken chiral symmetry of low-energy QCD [3].

Nuclear potentials based on chiral perturbation theory (ChPT) $[1,4,5]$ are at present largely employed so to bridge the QCD, that is the fundamental theory of strong interactions, to the physics of the atomic nucleus. A relevant apect of ChPT is that nuclear two-body forces, many-body forces, and currents $[1,6,7]$ are generated on an equal footing. As a matter of fact, some low-energy constants (LECs) which appear in the two-nucleon-force $(2 \mathrm{NF})$, fitted to two-nucleon data, belong also to the expressions of the threenucleon forces (3NF), four-nucleon forces (4NF), and electroweak currents.

In this contribution we will review about calculations for many-body systems, both finite nuclei and infinite nuclear matter, performed starting from nuclear forces derived within the ChPT. More precisely, we consider a lowmomentum chiral potential constructed at next-to-next-to-next-to-leading order $\left(\mathrm{N}^{3} \mathrm{LO}\right)$ using a sharp cutoff $\Lambda=414 \mathrm{MeV}$ [8], dubbed as $\mathrm{N}^{3} \mathrm{LOW}$.

The paper is organized as follows. In Sec. 2, we briefly outline the theoretical framework of our many-body calculations. More precisely, we will present a few details of the chiral potential we have employed, of the theory underlying the derivation of an effective hamiltonian for a shell-model calculation, and of the perturbative expansion of the energy per particle in infinite neutron- and symmetric-nuclear matter.

Sec. 3 is dedicated to the presentation of results of realistic shell-model calculation for medium-mass nuclei outside ${ }^{14} \mathrm{C}$ and ${ }^{16} \mathrm{O}$ closed-shell nuclei, starting from $\mathrm{N}^{3} \mathrm{LOW}$ chiral potential. The results of the calculation of the equation of state (EOS) for neutron- and symmetric-infinite matter are reported in Sec. 4, where we also focus on the comparison of the calculated symmetry energy with the empirical values.

Finally, in Sec. 5 our conclusions are reported.

\section{Outline of calculations}

First of all, we start this section with a brief description of the chiral potential $\mathrm{N}^{3} \mathrm{LOW}$. This potential has been introduced in Ref. [8], so to be compared directly with a $V_{\text {low-k }}$ derived from the chiral potential of Entem and Machleidt [4], renormalized within a cutoff momentum $\Lambda=2.1 \mathrm{fm}^{-1}$ [9]. 
In the $V_{\text {low-k }}$ approach, the original potential $V_{N N}$ is smoothed by integrating out the high-momentum modes of $V_{N N}$ down to a cutoff momentum $\Lambda$. This is achieved by a unitary transformation as the one, for example, suggested by Lee and Suzuki $[9,10]$.

Basically, the $V_{\text {low-k }}$ approach to nuclear structure physics is successful because the dynamics which rules nuclear physics can be described in the framework of a low-energy EFT.

As already mentioned in Sec. 1, this nuclear EFT is characterized by the spontaneously broken chiral symmetry, and the degrees of freedom which are relevant for nuclear physics, nucleons and pions.

In order to reach a proper convergence rate, the ChPT expansion is valid only for momenta $Q<\Lambda_{\chi} \simeq 1 \mathrm{GeV}$, where $\Lambda_{\chi}$ denotes the chiral symmetry breaking scale. Nucleon-nucleon $(N N)$ potentials derived from ChPT are typically multiplied by a (non-local) regulator function $f\left(p^{\prime}, p\right)=$ $\exp \left[-\left(p^{\prime} / \Lambda\right)^{2 n}-(p / \Lambda)^{2 n}\right]$, where $\Lambda \simeq 0.5 \mathrm{GeV}$ is a typical choice for the cutoff scale. In terms of a relative-momentum cutoff, this means that present chiral $N N$ potentials [4,5] typically apply values for $\Lambda$ around $2.5 \mathrm{fm}^{-1}$.

On these grounds, in Ref. [8] a chiral $\mathrm{N}^{3} \mathrm{LO} N N$ potential has been considered, using the regulator function in the above expression with $n=10$ and $\Lambda=414 \mathrm{MeV}$, so to be confronted directly with a $V_{\text {low-k }}$ whose cutoff momentum is $\Lambda=2.1 \mathrm{fm}^{-1}$. We have dubbed this potential $\mathrm{N}^{3} \mathrm{LOW}$.

One advantage of this potential, respect to a $V_{\text {low-k }}$ is that it is given in analytic form. The analytic expressions are the same as for the "hard" $\mathrm{N}^{3} \mathrm{LO}$ potential by Entem and Machleidt of 2003 [4].

The $\mathrm{N}^{3} \mathrm{LOW}$ potential reproduces accurately the empirical deuteron binding energy, the experimental low-energy scattering parameters, and the empirical phase-shifts of $N N$ scattering up to at least $200 \mathrm{MeV}$ laboratory energy (see fig. 1).

Another important issue are many-body forces. One great advantage of $\mathrm{ChPT}$ is that it generates nuclear two- and many-body forces on an equal footing. Most interaction vertices that appear in the $3 \mathrm{NF}$ and in the four-nucleon force (4NF) also occur in the two-nucleon force $(2 \mathrm{NF})$. The parameters carried by these vertices are fixed in the construction of the chiral $2 \mathrm{NF}$. Consistency requires that for the same vertices the same parameter values are used in the $2 \mathrm{NF}, 3 \mathrm{NF}, 4 \mathrm{NF}, \ldots$

If one considers the perturbative expansion of the $3 \mathrm{NF}$ at $\mathrm{N}^{2} \mathrm{LO}$, then only two extra-parameters need to be fixed, namely the low-energy constants (LECs) dealing with the one-pion exchange three-nucleon force $V_{3 N}^{1 \pi}$ and the contact three-nucleon force $V_{3 N}^{\text {cont }}$ at $\mathrm{N}^{2} \mathrm{LO}$. These 3NF LECs have been adjusted to $A=3$ observables, more precisely so to reproduce the ${ }^{3} \mathrm{H}$ 

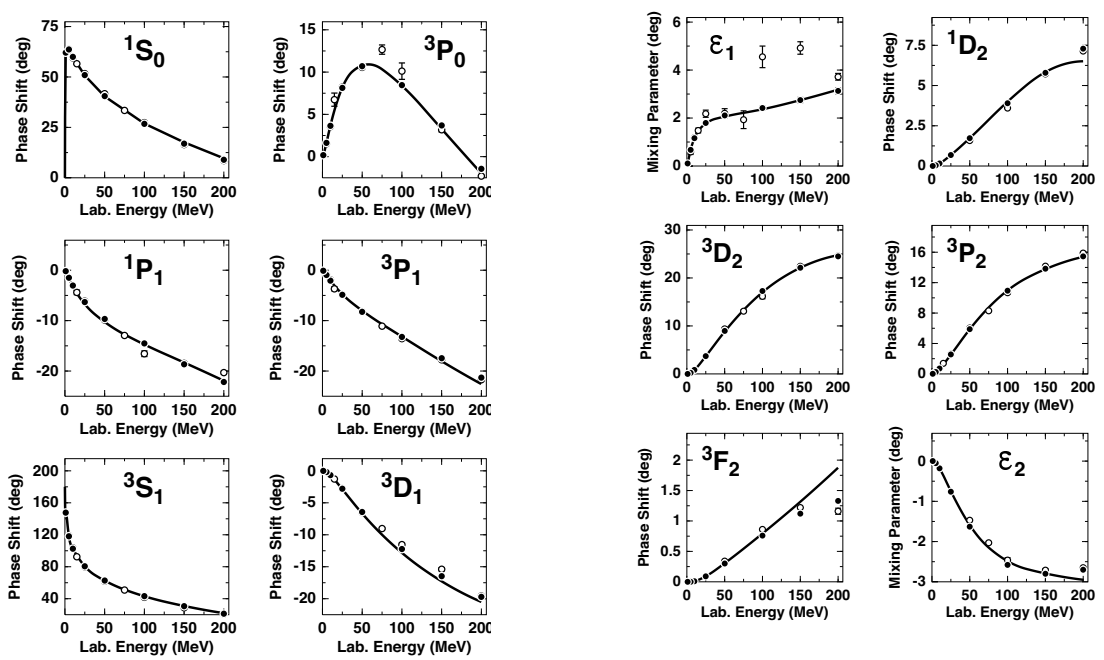

Figure 1: Phase parameters of neutron-proton scattering up to $200 \mathrm{MeV}$ laboratory energy. The solid lines show the predictions by the $\mathrm{N}^{3} \mathrm{LOW}$ potential. The solid dots and open circles represent the Nijmegen multienergy $n p$ phase shift analysis and the GWU/VPI single-energy $n p$ analysis SM99, respectively.

and ${ }^{3} \mathrm{He}$ binding energies together with the triton half-life (specifically the Gamow-Teller matrix element) $[11,12]$.

We have employed the $\mathrm{N}^{3} \mathrm{LOW}$ potential in calculations for both finite nuclei $[8,13,14]$ - within the framework of the realistic shell model - and for infinite nuclear matter $[12,15]$.

Let us start introducing the realistic shell-model.

We intend as a realistic shell-model, calculations for finite nuclei where the effective shell-model hamiltonian, namely the single-particle energies (s.p.e.) and two-body matrix elements (TBME) of the residual interaction, has been derived from a realistic $N N$ force [16], such as the $\mathrm{N}^{3} \mathrm{LOW}$ potential.

Starting from this potential, the shell-model effective hamiltonian is derived within the many-body perturbation theory, as developed by Kuo and coworkers through the 1970s [17]. More precisely, we have used the wellknown $\hat{Q}$-box plus folded-diagram method $[16,17]$, where the $\hat{Q}$-box is a collection of one- and two-body irreducible valence-linked Goldstone diagrams. In the following calculations the $\hat{Q}$-box includes all diagrams up to third order [16], and the folded-diagram series is summed up to all orders using the Lee-Suzuki iteration method [10]. We sum over a number of in- 
termediate states between successive vertices whose unperturbed excitation energy is less than a value $E_{0}=E_{\text {max }}$, which is sufficiently large to ensure that both s.p.e. and TBME are almost independent from the choice of $E_{\max }$.

As regards the calculation of EOS of infinite nuclear matter, we calculate the ground state energy (g.s.e.) per particle within the framework of manybody perturbation theory. More precisely, the g.s.e. is expressed as a sum of Goldstone diagrams up to third order.

The effects of the $3 \mathrm{NF}$ are taken into account via a density-dependent two-body potential $\bar{V}_{3 N}$, that is added to the chiral $\mathrm{N}^{3} \mathrm{LO}$ potential $V_{2 N}$. This potential is obtained by integrating one nucleon up to the Fermi momentum $k_{F}$, thus leading to a density-dependent two-nucleon interaction $\bar{V}_{3 N}\left(k_{F}\right)$. Presently, the analytic expressions for $\bar{V}_{3 N}[18]$ has been derived only for the $\mathrm{N}^{2} \mathrm{LO} 3 \mathrm{NF}$, which is the one we take into account in the calculation of our EOS. In order to consider the correct combinatorial factors of the normal-ordering at the two-body level of the $3 \mathrm{NF}$, the matrix elements of $\bar{V}_{3 N}\left(k_{F}\right)$ have to be multiplied by a factor $1 / 3$ in the first-order Hartree-Fock (HF) diagram, and by a factor $1 / 2$ in the calculation of the HF single-particle energies $[12,15]$.

The analytic expressions of first-, second-, and third-order particleparticle $(p p)$ and hole-hole $(h h)$ contributions, together with the one of single-particle HF potential, are reported in Ref. [15]. The implicit expression of the third-order particle-hole $p h$ diagram - also known as the ring diagram - can be found in Ref. [19].

\section{Shell-model results}

In Ref. [13], we have presented a shell-model description of heavy carbon isotopes, using a fully microscopic approach, starting from $\mathrm{N}^{3} \mathrm{LOW}$ potential and using ${ }^{14} \mathrm{C}$ as a closed core. As mentioned in sec. 2 , both the single-particle energies and residual two-body interaction have been derived within the many-body perturbation theory. Our calculations have led to a successful description of these isotopes when approaching the neutron dripline. More precisely, our results have reproduced the disappearance of the $N=14$ subshell closure that is present in the oxygen chain, and predicted the $N=16$ one.

Moreover, the shell-model calculations have predicted that ${ }^{21} \mathrm{C}$ is unstable against one-neutron decay, fitting the picture of ${ }^{22} \mathrm{C}$ as a Borromean nucleus. It is worth mentioning that the latter is one of the most exotic nuclei of the 3000 known isotopes, its $N / Z$ ratio being 2.67 , and can be 


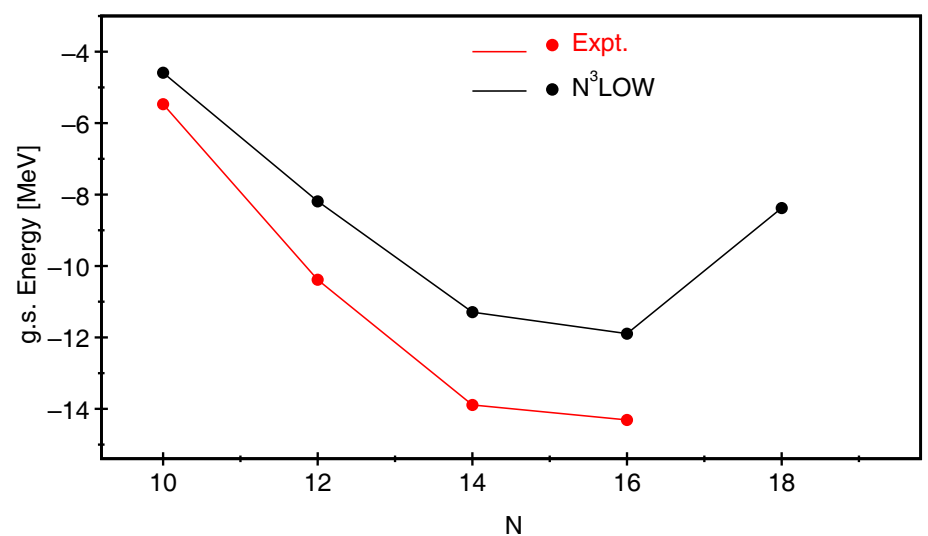

Figure 2: (Color online) Experimental and calculated ground-state energies for carbon isotopes from $A=16$ to $24 . N$ is the number of neutrons. See text for details.

considered the heaviest Borromean nucleus ever observed. We reproduce successfully the fact that ${ }^{22} \mathrm{C}$ is the last bound isotope (see fig. 2).

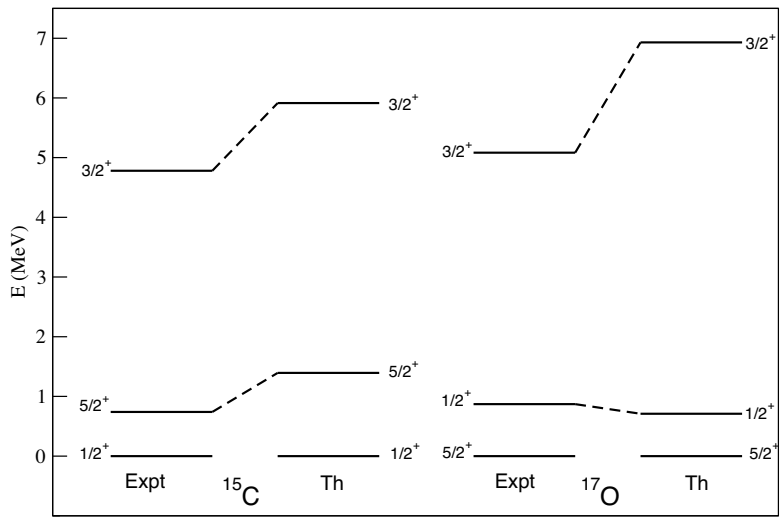

Figure 3: Experimental [20] and theoretical single-particle states of ${ }^{15} \mathrm{C}$ and ${ }^{17} \mathrm{O}$.

In ref. [13] we investigated only the identical-particle channel of the effective hamiltonian. Here, we point out that also the proton-neutron channel features well, as testified in fig. 3 where the experimental and theoretical single-particle spectra of ${ }^{15} \mathrm{C}$ and ${ }^{17} \mathrm{O}$ are reported. 


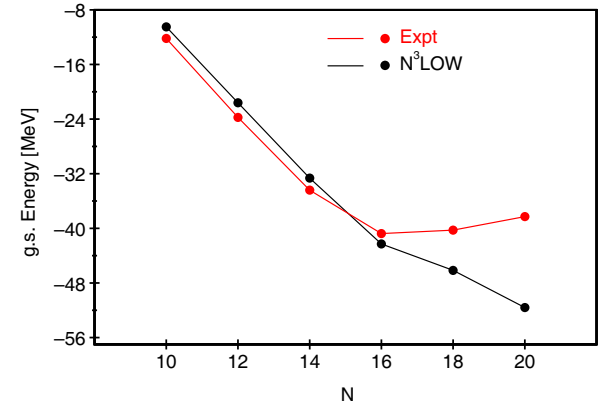

Figure 4: (Color online) Experimental and calculated ground-state energies for oxygen isotopes from $A=18$ to $24 . N$ is the number of neutrons.

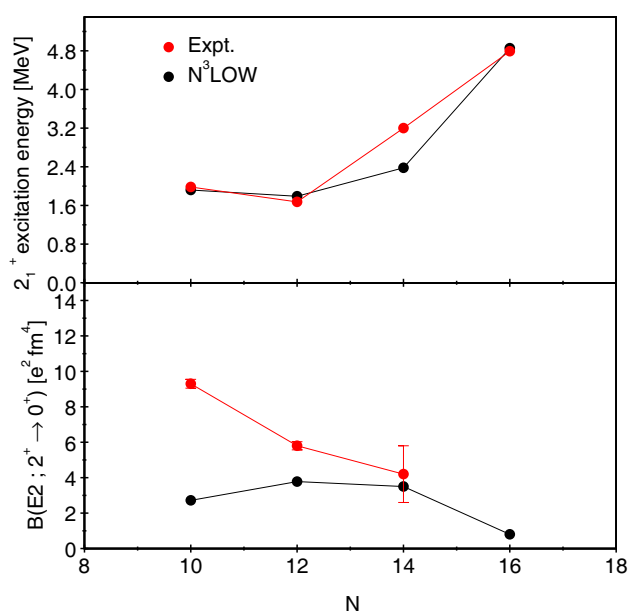

Figure 5: (Color online) Experimental [20] and calculated excitation energies of the yrast $J^{\pi}=2^{+}$states and $B\left(E 2 ; 2_{1}^{+} \rightarrow 0_{1}^{+}\right)$transition rates for oxygen isotopes.

As can be seen the inversion of the $J^{\pi}=1 / 2^{+}$and $5 / 2^{+}$states is correctly reproduced, thanks to the proton-neutron monopole component of the effective hamiltonian.

We have employed the $\mathrm{N}^{3} \mathrm{LOW}$ potential also for calculations of nuclei belonging to the $s d$-shell, using ${ }^{16} \mathrm{O}$ as a closed core. In ref. [23] we reported only results for two-valence-particle nuclei ${ }^{18} \mathrm{O}$ and ${ }^{18} \mathrm{~F}$. Here we report in fig. 4 the calculated ground-state energies, with respect to ${ }^{16} \mathrm{O}$, of even-mass oxygen isotopes and compare them with the experimental ones. As can be observed, the agreement is quite remarkable up to ${ }^{24} \mathrm{O}$, then the theory predicts a bound ${ }^{26} \mathrm{O}$, at variance with experiment. This probably traces back to the fact that we have not included the effect of three-body forces in the derivation of our effective hamiltonian [24]

In fig. 5 we have also reported the experimental and calculated excitation energies of the yrast $J^{\pi}=2^{+}$states and $B\left(E 2 ; 2_{1}^{+} \rightarrow 0_{1}^{+}\right)$transition rates.

\section{Infinite nuclear matter}

As mentioned previously, we calculate the energy per particle of infinite nuclear matter in the framework of the Goldstone expansion taking into account the effects of the $\mathrm{N}^{2} \mathrm{LO} 3 \mathrm{NF}$ via the introduction of a densitydependent two-body potential $\bar{V}_{N N N}\left(k_{F}\right)$. 


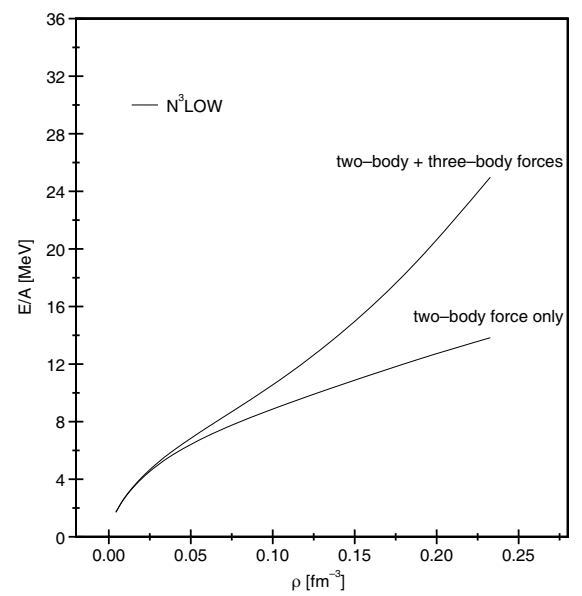

Figure 6: Results obtained for the PNM energy per nucleon, with and without $3 \mathrm{NF}$ effects.

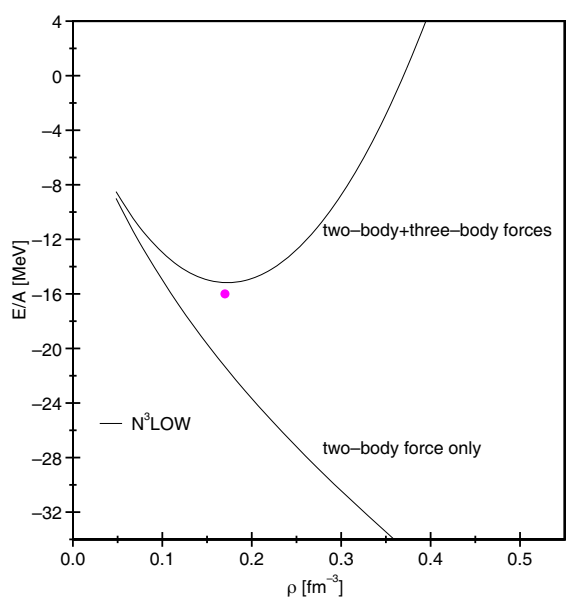

Figure 7: (Color online) Same as in Fig. 7, but for the PNM energy per nucleon.

In Fig. 6 and 7 we show, respectively, the pure-neutron matter (PNM) and symmetric-nuclear matter (SNM) EOS as a function of density, calculated with and without including the $3 \mathrm{NF}$ contributions.

From the inspection of the figures, it can be seen that the repulsive effect arising from $3 \mathrm{NF}$ is consistent in both cases. In particular it can be observed that the $3 \mathrm{NF}$ contribution to the energy per nucleon in SNM is larger than that in PNM, and that its inclusion proves to be crucial to reproduce the empirical saturation properties.

The symmetry energy $E_{\text {sym }}$ is defined as the strength of the quadratic term in an expansion of the energy per particle $(\bar{E})$ in asymmetric matter with respect to the asymmetry parameter $\alpha=\left(\rho_{n}-\rho_{p}\right) /\left(\rho_{n}+\rho_{p}\right)$ :

$$
\bar{E}(\rho, \alpha) \simeq \bar{E}(\rho, \alpha=0)+E_{\text {sym }} \alpha^{2}+\mathcal{O}\left(\alpha^{4}\right) .
$$

Many microscopic calculations provide a nearly linear behavior of $\bar{E}(\rho, \alpha)$ as a function of $\alpha^{2}$, and this justifies the common approximation of defining the symmetry energy as the difference between the energy per particle in PNM and SNM, neglecting powers beyond $\alpha^{2}$ in the $\bar{E}(\rho, \alpha)$ expansion. The density dependence of $E_{\text {sym }}$ can be correlated with the neutron skin thickness of nuclei and the radius of neutron stars, and systematic efforts are ongoing to get better empirical constraints on its value, through both laboratory and astrophysical measurements.

In Fig. 8, our results for the symmetry energy as a function of density are reported. In this connection, of particular interest is the slope of the 


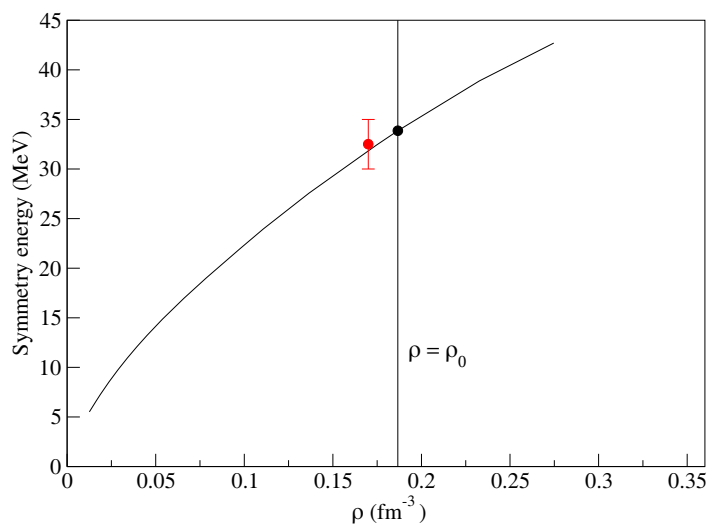

Figure 8: (Color online) Calculated symmetry energy as a function of density. The red error bar represents the empirical constraint on $E_{\text {sym }}[21]$.

symmetry energy at the saturation density $L$ defined as

$$
L=3 \rho_{0}\left(\frac{\partial E_{\text {sym }}}{\partial \rho}\right)_{\rho_{0}} .
$$

Our calculated value for $L$ is $68 \mathrm{MeV}$, that has to be compared with the not yet firmly constrained empirical value of $L=70 \pm 25 \mathrm{MeV}$ [22].

\section{Conclusions}

In this paper, we have presented nuclear structure calculations, based on the realistic $\mathrm{N}^{3} \mathrm{LOW}$ chiral potential, for both finite nuclei and infinite nuclear matter.

The quality of the results obtained for finite nuclei, and the ability to provide realistic nuclear matter predictions, makes us confident that the approach to nuclear structure, based on chiral potentials, may be a valuable tool to study properties of stable and unstable nuclear systems.

This excellent agreement paves the way towards an exciting future for the study of the physics of nuclei.

\section{References}

[1] R. Machleidt and D. R. Entem, Phys. Rep., 503 (2011) 1.

[2] E. Epelbaum, et al., Rev. Mod. Phys., 81 (2009) 1773. 
[3] S. Weinberg, Physica, 96A (1979) 327.

[4] D. R. Entem and R. Machleidt, Phys. Rev. C, 68 (2003) 041001(R).

[5] E. Epelbaum, et al., Nucl. Phys. A, 747 (2005) 362.

[6] S. Weinberg, Phys. Lett. B, 295 (1992) 114.

[7] U. van Kolck, Phys. Rev. C, 49 (1994) 2932.

[8] L. Coraggio, et al., Phys. Rev. C, 75 (2007) 024311.

[9] S. Bogner, et al., Phys. Rev. C, 65 (2002) 051301(R).

[10] S. Y. Lee and K. Suzuki, Phys. Lett. B, 91 (1980) 173.

[11] L. E. Marcucci, et al., Phys. Rev. Lett., 108 (2012) 052502.

[12] L. Coraggio, et al., Phys. Rev. C, 89 (2014) 044321.

[13] L. Coraggio, et al.,Phys. Rev. C, 81 (2010) 064303.

[14] L. Coraggio, et al., Nucl. Phys. A, 928 (2014) 43.

[15] L. Coraggio, et al., Phys. Rev. C, 87 (2013) 014322.

[16] L. Coraggio, et al., Ann. Phys., 327 (2012) 2125.

[17] T. T. S. Kuo and E. Osnes, Lecture Notes in Physics, 364 (1990).

[18] J. W. Holt, et al., Phys. Rev. C, 81 (2010) 024002.

[19] James J. MacKenzie, Phys. Rev., 179 (1969) 1002.

[20] Data extracted using the NNDC On-line Data Service from the ENSDF database, file revised as of September 30, 2015.

[21] M. Dutra et al., Phys. Rev. C, 85 (2012) 035201.

[22] M. B. Tsang et al., Phys. Rev. C, 86 (2012) 015803.

[23] L. Coraggio, et al., Nucl. Phys. A, 928 (2014) 43.

[24] T. Otsuka, Phys. Rev. Lett., 105 (2010) 032501. 